\title{
Teoriya Resheniya Izobreatatelskikh Zadatch (TRIZ) et Propriété Intellectuelle pour renforcer l'esprit start-up
}

\section{Teoriya Resheniya Izobreatatelskikh Zadatch (TRIZ) and Intellectual Property to strengthen the start-up spirit}

\author{
Yves Guillou ${ }^{1}$, Eric Prevost ${ }^{2}$, Alain Riwan ${ }^{3}$, Pascal Sire ${ }^{4}$ \\ ${ }^{1}$ Yves Guillou, Guilbert EXPRESS, Fontenay sous bois, yves.guillou@gmail.com \\ ${ }^{2}$ Eric Prevost, Oracle Corp \& Président TRIZ France, Paris, epbus@yahoo.fr \\ ${ }^{3}$ Alain Riwan, CEA, LIST, Interactive Robotics Laboratory, Gif-sur-Yvette, alain.riwan@cea.fr \\ ${ }^{4}$ Pascal Sire, Agence d'innovation ouverte OpenAl, Strasbourg, pascal.sire@openai.co
}

\begin{abstract}
RÉSUMÉ. Comment les start-ups peuvent-elles bénéficier des bonnes pratiques issues du croisement entre invention, innovation et propriété intellectuelle $(\mathrm{PI})$ afin de contribuer à cette réflexion vitale pour la survie de nos industries ? Les experts de l'association TRIZ France puisent dans leurs expériences concrètes pour illustrer les apports méthodologiques et pratiques de la Théorie de résolution des problèmes d'invention (TRIZ), avec un intérêt particulier pour les entreprises où « l'esprit start-up » permet de faciliter l'idéation et d'accélérer la production de produits et services innovants.

ABSTRACT. How can start-ups benefit from good business innovation practices while enforcing the link between invention, innovation and intellectual property (IP) in order to contribute to this vital discussion for the survival of our industries? Some experts of the association TRIZ France have drawn from their practical experiences to present a path to illustrate the methodological and practical contributions of the Theory for inventive problem solving (TRIZ). This illustration highlights a specific focus on businesses where the "start-up spirit" facilitates ideation and accelerates the production of innovative products and services.

MOTS-CLÉS. Conception inventive, propriété intellectuelle, start-up, TRIZ.

KEYWORDS. Innovation design, intellectual property, start-up, TRIZ.
\end{abstract}

\section{Introduction : la conception inventive et l'esprit start-up}

"Start-ups are only temporary organizations, designed to search - not execute- for a scalable and repeatable business model" [BLA 16]. "Une start-up est une phase temporaire vécue par un groupe de personnes qui testent des choses jusqu'à ce que cela fonctionne à petite échelle, puis qui passent à une phase de scaling afin d'en faire une grande entreprise" [BER 16]. Une start-up c'est "une institution humaine conçue pour créer un nouveau produit ou service dans des conditions d'incertitude extrême" [RIE 12]. Etre une start-up n'est pas une question d'âge, ni de taille, ni de secteur d'activité, mais doit répondre aux quatre conditions suivantes : forte croissance potentielle, utiliser une nouvelle technologie, avoir besoin de financement massif (fameuses levées de fonds) et être sur un marché nouveau dont le risque est difficile à évaluer [FRI 16].

L'esprit start-up, c'est donc un mode de fonctionnement qui stimule l'innovation, la créativité, peut être même rébellion et renforce l'esprit d'équipe, la spontanéité, le risque d'échouer, l'agilité et l'adaptation rapide [TRE 12].

IMPORTANT. « start-up = disruption, croissance et incertitude, et esprit start-up = esprit pionnier »

Comment s'inspirer de cet esprit pour redynamiser les entreprises plus traditionnelles, maintenant que toutes les entreprises souhaitent s'afficher innovantes ? Comment, dans un contexte de résolution de problèmes techniques, amener les ingénieurs à trouver des solutions innovantes et pertinentes de manière systématique, à partir de cet esprit ? La résolution de problèmes techniques de manière innovante (que nous allons assimiler à la conception inventive) est une démarche intellectuelle 
indépendante du domaine technique du problème à résoudre et des disciplines concernées par les solutions [TRI 16a] :

- Les principes de résolution sont en nombre fini et ont été répertoriés;

- Les inventions répertoriées dans les brevets ont été classées selon différentes taxonomies ;

- La définition fonctionnelle des objets techniques évolue dans le temps selon une logique commune à tous, logique caractérisée selon huit tendances.

En tant que résolution innovante de problème technique, la démarche d'innovation peut donc s'apprendre, sur la base de deux notions fortes :

- La résolution d'un problème technique doit se situer dans une démarche d'optimisation globale conservant la satisfaction des fonctions techniques précédemment opérées par l'objet technique et accueillant une caractéristique fonctionnelle nouvelle sans pénaliser les caractéristiques d'origine. Résoudre un problème technique dans une perspective d'évolution de fonctionnalités techniques de cet objet consiste donc à trouver une solution élégante qui permettra de faire véritablement disparaitre les incompatibilités fonctionnelles (que nous décrirons plus loin sous le terme de "contradictions") afin d'améliorer globalement le résultat attendu de l'objet technique aux fonctionnalités augmentées ;

- Toute solution innovante augmente donc le portefeuille de fonctionnalités de l'objet technique (ce que nous nommerons idéalité du système, afin de bien marquer la finalité de l'évolution fonctionnelle, en interprétant cette notion comme un ratio fonctionnalité sur (parasites plus coût). L'idéalité ainsi définie se rapproche de l'esprit de "faire mieux en consommant moins de ressources et de temps", propre à l'innovation frugale.

L'objet de cet article est donc d'instruire l'analyse d'une méthodologie apte à résoudre des problèmes techniques bloquant l'évolution fonctionnelle d'un objet technique, de telle sorte à faire progresser ce dernier vers l'idéalité par optimisations successives inscrites dans des trajectoires d'innovation qu'il va s'agir d'identifier, puis d'instruire l'inscription de cette méthodologie dans des stratégies d'acteurs (incubateurs et start-ups, entreprises innovantes) et d'instruire enfin la diffusion de cette méthodologie de conception inventive en la sortant du cadre des procédures industrielles conventionnelles pour la faire prospérer dans l'esprit start up en tant qu'application de management de l'innovation.

Cette méthodologie, qui n'est autre que celle associée à TRIZ, consiste en une approche heuristique destinée à la résolution de problèmes techniques élaborée sur la base des postulats de l'évolution des systèmes techniques [TRI 16b], postulats issus de la compilation d'un grand nombre de brevets d'invention. TRIZ est donc une méthode systématique de résolution créative de problème, intense en connaissances et exploitant le principe d'analogie [LOU 08], principe qui suggère d'explorer des solutions génériques, empruntées à d'autres domaines, non encore appliquées au problème particulier concerné. L'importance de TRIZ au sens des techniques de résolution de problèmes provient de sa nature de méta-méthodologie déployable dans de nombreux domaines de connaissances: en effet, TRIZ regroupe des orientations de raisonnement aptes à structurer un procédé mental de résolution de problème, lequel procédé est instrumenté par ARIZ, qui en constitue l'instanciation opérationnelle dans son acception proprement méthodologique [SAU 13].

Le présent article va débuter en situant la méthodologie de résolution de problèmes associée à TRIZ comme un phénomène majeur de l'histoire des méthodes scientifiques $(\S 1)$. Le paragraphe 2 s'attachera à mettre au jour les principaux éléments constituant le socle du cheminement mental de la méthodologie dont le fil conducteur est constitué par des postulats de l'évolution des systèmes techniques, à laquelle est adossé le principe d'analogie utilisé par TRIZ. Dans le paragraphe 3, il s'agit d'exploiter les postulats des tendances d'évolution pour mettre en action le principe d'analogie. Les 
éléments constituant le socle du cheminement mental propre à TRIZ présentés dans le paragraphe précédent peuvent maintenant être articulés en une méthodologie. Le paragraphe 4 a pour objet d'illustrer les considérations précédentes par des études de cas consacrées à un environnement de groupe industriel ou de start up. La vocation du paragraphe 5 consiste à discuter la possibilité pour l'article d'avoir épuisé le sujet de l'apport potentiel de la méthode TRIZ aux entrepreneurs de start up. La conclusion fait la synthèse des leçons apprises, des limitations et des perspectives de l'article.

\section{Cadre historique de TRIZ}

L'objet du présent cadre historique est de situer TRIZ comme un phénomène de pensée technique né au milieu du $20^{\text {ème }}$ siècle, toujours d'actualité deux générations plus tard, consacré à l'approche méthodologique de l'invention technique envisagée comme une méthode de créativité dans le cadre de résolution inventive de problèmes techniques.

TRIZ, inventée par Altshuller, est l'acronyme russe d'une expression (« Teoriya Resheniya Izobreatatelskikh Zadatch ») signifiant " Théorie de la résolution des problèmes inventifs ». C'est un ensemble de méthodes et d'outils, originellement associé aux techniques des années 1950-1960, dont l'objectif est d'aider à résoudre les problèmes techniques en systématisant l'identification des modèles de solutions et des procédés intellectuels de résolution inventive de problème.

\subsection{De l'analyse systématique des brevets d'invention à des principes de conception inventive}

L'élaboration de TRIZ s'est déroulée en plusieurs étapes et plus de deux millions de documents de brevet ont été analysés pour en extraire des principes d'innovation. De cette analyse est sortie la confirmation que l'information en matière de brevets constitue une source d'information extrêmement utile pour l'apprentissage et le développement de stratégies en matière de résolution des problèmes et d'innovation [WIP 16].

Pour faire émerger les principes de résolution inventive de problèmes, Althusser a bâti une méthodologie (baptisée ARIZ, pour Algorithme de résolution des problèmes d'invention) mettant en œuvre de nombreux outils, allant du déblocage de l'inertie psychologique qui freine les ingénieurs à la résolution des incompatibilités (dénommées contradictions) à la génération de solutions partielles.

\subsection{Vers une méthode pour apprendre à inventer}

Pour lui, l'enjeu consiste en effet à répondre à des questions telles que « comment faire léger et résistant à la fois ? » ou encore « comment être dur à un instant et mou à un autre ? ». Or l'innovation apparait souvent lorsque l'on sait résoudre cette contradiction. La méthode conduit l'ingénieur à exprimer son problème sous forme de contradictions élémentaires, qui peuvent alors être résolues avec une matrice, laquelle renvoie vers les principes qui, par le passé, ont déjà résolu cette contradiction dans d'autres contextes techniques.

Pourquoi est-il si difficile d'inventer? La difficulté d'inventer vient souvent des limitations des ressources (temps, argent, quantité de travail,...) ou des connaissances (théoriques, pratiques, organisationnelles), mais surtout de la capacité de l'inventeur à contourner son inertie psychologique. TRIZ demande une manière de penser détachée du problème industriel pouvant puiser dans des modèles de solutions de disciplines différentes et une certaine maitrise de la mise en pratique de ses outils. Dans une structure dédiée à la recherche d'innovation par méthodes de résolution de problème, la mise en place d'un processus de recherche de solutions innovantes intégrant TRIZ dans son ensemble est nécessaire pour être vraiment efficace [MAR 16]. 


\subsection{Des outils largement déployés dans l'industrie}

Les outils de TRIZ sont utilisés en France particulièrement dans l'industrie automobile et dans l'aéronautique, mais des applications à d'autres secteurs sont fréquentes [LEU 07]. Ils permettent à la fois de résoudre des problèmes d'inventivité, de préparer des dépôts de brevets mais aussi d'initialiser la mise en place des stratégies de $R \& D[D U B$ 09].

L'exploitation de la ressource méthodologique en matière de créativité explique le formidable engouement des entreprises occidentales pour TRIZ à partir des années 1990. Boeing, Procter \& Gamble, Eli Lilly, Ford, Agilent Technologies et bien d'autres, se sont faits les chantres de la méthode aux Etats-Unis. En France, le fabricant de cosmétiques Bourjois, l'électrotechnicien Legrand, les équipementiers MGI Coutier et la filiale française d'ArvinMeritor, figurent parmi ses utilisateurs réguliers, PSA jouant le rôle de chef de file. Depuis 1998, le constructeur automobile français a en effet mené seize études avec la méthode ou les logiciels affiliés, en mécanique comme en carrosserie. Elles ont débouché sur quarante-trois solutions applicables et neuf dépôts de brevets [USI 03].

TRIZ est à l'innovation ce que Lean et SixSigma sont à l'amélioration continue et, de même que Toyota est la référence mondiale Lean, Samsung est devenue rapidement la référence mondiale TRIZ. Sa croissance exceptionnelle d'innovation et de performance économique et commerciale a permis au conglomérat sud-coréen de devenir en quelques années un des leaders dans de nombreux domaines (électronique, électroménager,...), en grande partie par la mise en œuvre systématique de TRIZ depuis 2007 dans toutes les couches de son organisation, comme méthode principale d'innovation d'entreprise, ce qui lui a permis de réaliser une forte croissance de son chiffre d'affaire (de 162,9 $\mathrm{m} \$$ à $220,1 \mathrm{~m} \$$ entre 2006 et 2011).

D'autres grands groupes utilisent aujourd'hui massivement TRIZ pour accroître l'efficacité de leurs démarches d'innovation (Siemens, General Electric,...). La démarche TRIZ est ainsi mondialement reconnue, pragmatique et efficace pour le développement de l'innovation en entreprise et, comme Lean et SixSigma, elle requiert une approche d'entreprise rigoureuse et répétitive pour être efficace.

L'association TRIZ européenne (ETRIA) a mis en place en 2009 une enquête ayant pour but de mesurer l'état de diffusion de TRIZ dans le monde. Les résultats montrent que les entreprises appliquant TRIZ ont observé un gain dans la quantité d'idées mais aussi dans la qualité des idées émises. Il ressort aussi de l'étude que les concepts inventifs ayant pour origine TRIZ sont souvent sujets à être brevetés. De même, la majorité des sociétés ayant expérimenté TRIZ, souhaite mettre en place son déploiement en interne [CAV 12].

IMPORTANT. «L'inventivité et la créativité peuvent être apprises. » (G. S. Altshuller)

\section{Cadre théorique}

L'objet du paragraphe 2 est d'introduire le fil conducteur des postulats de l'évolution des systèmes techniques, à laquelle est adossé le principe utilisé par TRIZ, le principe d'analogie. Ce paragraphe débutera par quelques définitions spécifiques, puis il discutera la possibilité de qualifier ou non TRIZ de théorie. Il déroulera ensuite le principe de l'approche de résolution inventive de problème (chercher le "Pourquoi ?" plutôt que le "Comment ?", investiguer les tendances d'évolution, caractériser leur caractère linéaire ou non), afin de munir le lecteur des éléments constituant le socle du cheminement mental propre à TRIZ.

\subsection{Définitions}

La méthodologie TRIZ étant adossée à un formalisme poussé, il est indispensable de préciser certaines définitions dans l'acception qui lui est propre. 


\subsubsection{Situation initiale et énoncé du problème technique}

Une situation initiale est associée au dysfonctionnement d'un système qu'il s'agit d'éliminer ou à la nécessité d'améliorer une technique [LOU 08].

\subsubsection{Système technique}

Il s'agit d'une notion qui « a pour ambition d'exprimer l'interdépendance étroite qui relie entre elles les différentes composantes de la technologie à un moment donné de l'histoire » [CAR 97].

\subsubsection{Techniques}

Le développement du système technique et du processus technologique est souvent similaire et ils partagent un certain nombre de propriétés communes, qui permettent de les considérer comme un groupe unifié: la technique [LOU 08].

\subsubsection{Fonctions}

Une technique peut exécuter diverses fonctions, parmi lesquelles la fonction principale qui porte en elle la finalité de la technique. Des fonctions auxiliaires accompagnent et éclairent l'exécution de la fonction primaire : les fonctions utiles, les fonctions nuisibles, les fonctions neutres [LOU 08].

\subsubsection{Contradictions}

Les contradictions ou contraintes de conception, sont de trois types : administratives, techniques et physiques [LOU 08].

\subsubsection{Résultat final idéal}

A la base de TRIZ figure le postulat formulé par Altshuller selon lequel tous les systèmes technologiques évoluent vers un idéal. La progression vers cet idéal est mesurée par le ratio de la somme des fonctions utiles à la somme des fonctions nuisibles, qui peut être vu comme un taux d'efficacité intrinsèque. Le système technique idéal apparaît alors comme ayant un taux d'efficacité maximal. A travers le concept de résultat final idéal, c'est l'inertie psychologique inhérente au comportement humain en situation d'invention qui est visée, de sorte que toute possibilité de compromis par une relaxation des contraintes est exclue. Le résultat final idéal supprime les inconvénients et conserve les avantages, rend le système plus aisé à la manipulation, utilise les ressources disponibles, n'introduit pas de nouveaux inconvénients et fait évoluer le système technique vers un niveau de qualité de plus en plus élevé. Il se concentre sur les attentes et besoins du client ou sur les fonctions que le système doit accomplir, mais non sur le processus et les équipements existants [LOU 08].

\subsubsection{Ressources}

Dans TRIZ classique, le concept de ressources fait référence essentiellement au système technique et à son environnement, de même que l'apparition d'un problème est considérée comme due à l'indisponibilité d'une ressource nécessaire à l'accomplissement d'une ou plusieurs fonctions du système technique [LOU 08].

\subsubsection{TRIZ : théorie ou technique?}

Le fait de présenter TRIZ soit comme une théorie soit comme une technique sépare en deux clans l'abondante littérature consacrée à TRIZ. D'après [SAU 13], TRIZ est avant tout la démarche empirique d'une technique de créativité appliquée au cadre du problem solving, adossée à une formalisation méthodologique et instrumentée par un ensemble d'outils. Cinquante ans après les premiers travaux de Genrich Altshuller, la démarche TRIZ a été théorisée comme un élément de la 
procédure globale de résolution de problème visant à traduire la logique des transitions s'opérant entre les contradictions [LOU 08]. Cette logique qui débute par la formulation de l'énoncé du problème, puis qui est progressivement construite au fil de l'examen des divers types de contradictions, inclut, entre autres, la méthodologie TRIZ, mais également la Theory of Constraints Thinking Process Approach [SAU 13]. Le principe de base de la méthode TRIZ est le principe d'analogie, utilisé en s'inspirant de solutions obtenues dans d'autres domaines techniques, mais le principe d'analogie n'est pas lui-même théorisé dans la méthode [SAu 13].

\subsection{TRIZ, aide à la conception inventive ou "Pourquoi ?" plutôt que "Comment ?"}

Selon Altshuller, la stratégie de résolution de problèmes doit graduellement réduire l'aire de recherche au lieu d'accroître le nombre de variantes : c'est l'objectif affirmé de la méthode TRIZ, démarche convergente qui permet de reformuler le problème pour mieux le résoudre. Cette démarche est présentée à travers des exemples développés (et brevetés) au Laboratoire de Robotique Interactive, comme la roue omnidirectionnelle ou le bras robotisé gonflable, plus loin, plus léger, plus facile à mettre en œuvre et bien moins cher [VOI 12].

Pour l'expérimenter avec succès, il s'agit de se jeter à l'eau pour « inventer en 5 minutes » et rendre « indolore le bassin de plongée » [RIW 15], afin d'apprendre comment une chaîne de vélo est à la fois rigide (pour transférer l'énergie) et flexible (pour s'enrouler autour du plateau), comment la surface portante des ailes d'avion de chasse peut être grande pour des décollages courts et petite à grande vitesse, mais aussi comment «TRIZ aide les non-voyants à trouver leur chemin dans les stations de métro » [RIW 13] ou comment « améliorer l'expérience et la sécurité avec des robots interactifs » [RIX 14]. Toujours selon cet auteur, la production d'idées inventives est le point de départ de l'innovation, si nécessaire dans le contexte économique actuel et l'obligation de résultats impose la mise en œuvre d'un processus fécond et rationnel d'émergence d'idées en rupture, aptes à mieux répondre aux problèmes actuels et futurs [RIW 15].

IMPORTANT. Pour systématiser le processus de recherche de concepts, d'invention et de création de valeur brevetable, il est nécessaire de comprendre POURQUOI (il y a un problème) plutôt que tout de suite chercher COMMENT (trouver la solution).

Le Comment est une question "réflexe", alors que le Pourquoi est une question "réfléchie" [GUI 12].

\subsection{TRIZ et les tendances d'évolution}

Considérant qu'il vaut mieux résoudre la contradiction d'un problème sur la base du "Pourquoi ?" plutôt que du "Comment ?" [TRI 16a], TRIZ considère que les problèmes rencontrés durant la conception d'un nouveau modèle d'affaires, produit ou service présentent des analogies avec d'autres et que des solutions analogues doivent pouvoir s'appliquer [ALT 06]. Ce constat résulte de l'analyse d'un grand nombre de brevets menée par Altshuller [MEY 07].

\subsubsection{Les postulats fondateurs issus de l'analyse systématique des brevets d'invention}

Le résultat de l'analyse systématique des brevets a permis de mettre en évidence trois postulats [TRI $16 \mathrm{~b}]$, sur lesquels va être adossé le principe d'analogie :

- Les solutions sont analogues dans la majorité des secteurs industriels et scientifiques ;

- Les modèles d'évolution technique sont répétés dans la majorité des secteurs industriels et scientifiques ;

- Les innovations peuvent mettre en application des effets scientifiques issus d'autres domaines techniques. 
Ces constatations ont amené Altshuller à formaliser sa démarche de résolution des problèmes inventifs, sans résoudre directement le problème initial, mais en passant par la construction d'un problème générique à partir du problème initial. On obtient ainsi, par abstraction et sous forme de contradictions, un problème qui est indépendant du domaine technique du problème initial, ce qui permet ainsi de faire appel pour sa résolution à des effets scientifiques en dehors du domaine technique initial.

\subsubsection{Les hypothèses portées sur les tendances générales d'évolution}

Les huit tendances d'évolution sont définies comme suit [TRI 16a] :

- \#1 : Définition des conditions nécessaires et suffisantes à l'apparition d'un nouveau système viable

- \#2: Circulation correcte des flux énergétiques à travers le système technique

- \#3 : Coordination du rythme des parties pour un travail de concert

- \#4 : Tendance à l'augmentation du niveau d'idéalité

- \#5 : Absence de maillon faible dans les différentes parties

- \#6 : Tendance à l'intégration dans un système d'ordre supérieur

- \#7 : Transition de chaque moyen du niveau macroscopique vers le niveau microscopique

- \#8 : Adaptation aux contraintes extérieures

Depuis toujours, les inventeurs successifs ont semblé s'inspirer de leurs prédécesseurs (améliorations mineures par itérations successives), mais ont parfois apporté des éléments de rupture. Aujourd'hui, nous sommes surpris de constater que certaines inventions du passé auraient pu être plus rapides, juste en regardant ce qui se passait au même moment dans un autre domaine ou dans un autre pays.

« Pourquoi chercher à réinventer la roue alors que la solution existe déjà ? » (Altshuller)

\subsection{De la draisienne au vélo moderne, exemple universel pour comprendre l'évolution}

1817 : le baron Carl von Drais "inventa" la draisienne permettant de "courir assis" en soulageant l'utilisateur du poids du corps, avec un premier record de vitesse de 14,4 km/h. L'objet-fut malgré tout peu utilisé, car il était peu pratique.

1860 : Pierre Michaux, serrurier, lui apporta une évolution majeure : l'ajout de deux pédales sur la roue avant, ainsi qu'un frein et qu'une selle réglable. Les pédales permettaient de reposer les pieds et d'entraîner la roue. Dès lors, le vélocipède devint beaucoup plus populaire et commença à être produit industriellement.

1870 : L'évolution vers le grand-bi a été provoquée par la recherche de vitesse. Plus la roue est grande et plus la démultiplication est importante, mais elle oblige à se percher en hauteur. Le dimensionnement du grand-bi a fini par se stabiliser à la dimension maximale imposée par la longueur des jambes de l'utilisateur.

1885 : John K. Starley déposa le brevet de la "bicyclette de sécurité", qui comportait un pédalier, non plus sur la roue avant, mais sur le cadre, avec une transmission par chaîne sur la roue arrière. Traiter le problème induit par la grande roue et l'instabilité qu'elle provoquait, permit donc d'obtenir à cette étape le prototype de la bicyclette moderne.

D'autres créations verront le jour qui participeront à la diversité actuelle, mais il faudra attendre les années 1970 pour voir apparaître le VTT, soit un peu plus de 150 ans après la découverte du baron von Drais. 
Ce raccourci historique nous offre plusieurs constats :

- Tout d'abord, certaines améliorations-existaient déjà dans un autre domaine technique : le pédalier n'est rien d'autre que le dispositif utilisé par les rémouleurs pour faire tourner leur meule ;

- La conception est souvent limitée par les ressources de l'époque : la draisienne était en bois, car les techniques d'alors pour construire les chariots et voitures à bras utilisaient ce matériau. Ce que nous appelons aujourd'hui la technologie évoluera ensuite pour réaliser des composants métalliques moins massifs et plus adaptés au besoin ;

- L'évolution des fonctionnalités de l'objet technique est bloquée par l'une de ses fonctions. Il n'était plus possible d'augmenter diamètre de la roue du grand-bi et il a fallu une discontinuité (séparer le pédalier de la roue) pour pouvoir faire évoluer les performances et le confort d'utilisation ;

- La croissance des fonctionnalités de l'objet technique se décompose en plusieurs parties : un saut (l'invention du pédalier, par exemple) puis des phases d'optimisation (la taille de la roue du grand-bi) ;

- Les améliorations apportées ont donné naissance à une multitude d'objets, qui continuent à se diversifier et avoir eux-mêmes leur propre trajectoire évolutive. Chaque objet existant aujourd'hui a sa raison d'exister pour avoir amélioré telle ou telle fonction plutôt qu'une autre. Le VTT a évolué à partir du vélo de route et les deux sont aujourd'hui très différents, remplissant une fonction différente.

Voilà, en raccourci, les 150 ans d'amélioration d'un objet, avec ses nombreuses variantes. Certaines des améliorations existaient lors de la création de la draisienne (le pédalier par exemple, ainsi que le frein à sabot), mais il a tout de même fallu plus de 40 ans pour les intégrer à cet objet. L'histoire est ainsi parsemée d'exemples de cheminement sinueux de l'amélioration fonctionnelle, notamment parce que la recherche se fait par « essais et erreurs », centrés sur les techniques existantes et sans mettre en place une véritable organisation de ce que nous appelons aujourd'hui la R\&D. Sans certains de ces inventeurs, combien de temps au total aurait pris la stabilisation des améliorations de la bicyclette ?

\section{Cadre méthodologique}

L'objet du paragraphe 3 est d'exploiter les postulats des tendances d'évolution pour mettre en action le principe d'analogie. Les éléments constituant le socle du cheminement mental propre à TRIZ présentés dans le paragraphe précédent peuvent maintenant être articulés en une méthodologie. Il s'agit donc de repérer ces éléments, constitués par les tendances d'évolution, les analyser pour leur donner un sens prédictif au regard du déroulement du temps envisagé comme un élément de maturation fonctionnelle et technique.

\subsection{Observer les tendances d'évolution pour les anticiper}

Selon les postulats émis par Altshuller, la majorité des systèmes techniques de notre environnement semblent évoluer en suivant les tendances déjà mentionnées ainsi que celles détaillées juste après, qui chacune décrivent un comportement particulier dans l'évolution du système technique, lié à sa maturité et à sa position sur la « courbe en $\mathrm{S}$ » (figure 1), qui permet de le visualiser. Pour distinguer la première classe de tendances de la seconde, nous appellerons celles-ci les tendances caractérisées par la courbe en $\mathrm{S}$ et celles-là les tendances d'évolution générales.

L'axe horizontal de la figure 1 représente le temps et l'axe vertical, variable, représente un facteur lié à la performance du système technique. Ce facteur peut, par exemple, représenter des quantités produites de l'objet technique considéré. Cette définition implique que l'interprétation de la figure 1 va dépendre de la nature de la quantité portée en ordonnée.

Cette « courbe en $\mathrm{S} »$ est décomposée en trois grandes parties [GUI 11a] : 
- La première partie, infléchie vers le haut, représente le début de la vie de l'objet technique, considéré comme système technique, jeune et très perfectible et dont les améliorations sont rapides. Pendant cette période, le système technique améliore sa contrôlabilité, ses différentes parties s'ajustent et tendent à améliorer leur synchronisation ;

- La seconde partie, pratiquement linéaire, correspond à l'optimisation du système technique, qui tend à aboutir à une synthèse, par simplifications successives. A l'issue de cette période, le système technique est quasi-optimisé et peu d'éléments peuvent encore être améliorés de manière significative. En revanche, des disparités apparaissent entre ses composantes et certaines parties du système technique limitent l'amélioration de celui-ci.

- La troisième partie de la courbe, le haut du $\mathrm{S}$, correspond à la fin de l'évolution du système technique. Des améliorations apparaissent encore, mais de façon moins marquée. Le système technique est mature et se stabilise à un optimum.

La véritable découverte qui ressort de ces observations par l'analyse systématique des brevets d'invention est que ces tendances de courbe en $\mathrm{S}$ se répètent fortement, c'est-à-dire que le système technique évolue invariablement en les suivant. Bien sûr, des contre-exemples existent, qui semblent montrer que les "exceptions" à ces tendances ne résistent pas bien longtemps à la pression concurrentielle.

Les tendances d'évolution caractérisant la courbe en S sont habituellement présentées sous la forme de trois groupes [GUI 11b], elles ont chacune un rôle bien spécifique et sont appliquées en fonction du stade d'évolution du système technique :

- Les trois tendances statiques se situent au début de la courbe en S. Le système technique balbutie et cherche à remplir sa fonction principale tout en ajustant et en harmonisant le fonctionnement de ses divers composants.

- Les trois tendances cinématiques accompagnent le système technique dans son évolution sur la courbe en $\mathrm{S}$. Le rendement du système s'améliore, les sous-systèmes s'harmonisent et le système technique peut dans certains cas effectuer une transition vers l'intégration à un système technique d'ordre supérieur (appelé super-système).

- Les tendances dynamiques sont les dernières à être suivies avant le changement de courbe. Le système technique devient de plus en plus contrôlable : de nouveaux systèmes techniques font leur apparition, tendant à supplanter le système technique existant. Ceux-ci sont en rupture avec le système technique initial et sont, quant à eux, au début de leur « courbe en $\mathrm{S}$ ». La « courbe en $\mathrm{S}$ » d'un objet technique aura une période de développement plus courte que celle de l'objet technique précédent. 


\begin{tabular}{|c|c|c|}
\hline LOIS STATIQUES & LOIS CINÉMATIQUES & LOIS DYNAMIQUES \\
\hline $\begin{array}{c}\text { 1. Intégralité : } \\
\text { Le système } \\
\text { doit être complet } \\
\text { 2. Conductibilité } \\
\text { de l'énergie: } \\
\text { La chaîne de l'énergie } \\
\text { du système } \\
\text { doit être complète } \\
\text { 3. Coordination } \\
\text { du rythme: } \\
\text { Les différentes parties } \\
\text { du système doivent être } \\
\text { coordonnées }\end{array}$ & $\begin{array}{l}\text { 4. Accroissement } \\
\text { de l'idéalité : } \\
\text { L'utilité du système } \\
\text { augmente plus vite que } \\
\text { ses inconvénients } \\
\text { 5. Développement inégal: } \\
\text { Les différents constituants } \\
\text { du système evoluent } \\
\text { différemment } \\
\text { 6.hansition vers } \\
\text { le supersystème: } \\
\text { Le système ne peut plus } \\
\text { évoluer. Il poursuit son } \\
\text { développement comme } \\
\text { élément d'un système } \\
\text { plus complet }\end{array}$ & $\begin{array}{l}\text { 7. Transition } \\
\text { du niveau macro vers } \\
\text { le niveau micro: } \\
\text { La fonction utile } \\
\text { du système } \\
\text { est réalisée au niveau } \\
\text { microscopique } \\
\text { ou moléculaire } \\
\text { 8. Accroissement } \\
\text { du dynamisme } \\
\text { du système } \\
\text { Le système devient } \\
\text { plus souple, plus fluide. } \\
\text { II est plus facilement } \\
\text { contrôlable }\end{array}$ \\
\hline \multirow[t]{2}{*}{ NAISSANCE } & CROISSANCE & MATURITÉ \\
\hline & & Temps \\
\hline
\end{tabular}

Figure 1. TRIZ - Tendances d'évolution [SER 11]

\subsection{L'évaluation de la maturité du système technique au travers des tendances d'évolution}

Les tendances d'évolution générales ont donc un formidable outil pour faire de l'analyse comparative de produits, de fonctions, de systèmes... pour qui sait lire ce que ces courbes racontent et suggèrent [GUI 14].

Tout d'abord, leur utilisation permet d'estimer le positionnement du système technique analysé à travers des critères propres à chacune des huit tendances générales, en s'appuyant sur la notion de point d'observation, c'est-à-dire sur une analyse plus approfondie qu'une simple intuition. Ainsi, l'analyse du système en fonction de la première tendance générale [\#1 : Définition des conditions nécessaires et suffisantes à l'apparition d'un nouveau système viable)] permet de vérifier que rien ne manque pour réaliser la fonction principale, alors que la sixième tendance générale [\#6 : Tendance à l'intégration dans un système d'ordre supérieur)] permet de se demander dans quel super-système le système peut migrer et à quelles conditions.

Le système technique étudié peut donc être examiné par les tendances générales, en s'appuyant sur des points de repère, parmi lesquels :

- Le système technique précédent que l'on souhaite faire évoluer ;

- Les produits de la concurrence remplissant une fonction analogue ;

- Le système « idéal » au regard de la tendance générale examinée ;

- Des systèmes techniques issus d'autres domaines techniques, satisfaisant mieux certaines tendances générales, de façon potentiellement transposable ;

- La nature (bio-mimétisme) ...

Enfin, une fois les différents critères d'évaluation établis, il est intéressant de pouvoir regrouper ces résultats sous forme graphique, tout en gardant à l'esprit la maturité du système :

- Synthétiser les résultats obtenus sur un seul schéma ; 
- Suivre les évolutions au cours de la conception;

- Prédéterminer des schémas types de produit;

- Et piloter les évolutions du système, éventuellement sur plusieurs générations.

\section{Etudes de cas}

Il s'agit maintenant d'illustrer les considérations précédentes par des études de cas consacrées à un environnement de groupe industriel ou de start up.

\subsection{TRIZ et PI pour renforcer l'esprit start-up dans un monde concurrentiel}

En diffusant la vision de son comité «Industrie du futur » sur la transformation numérique de l'industrie, qu'il considère comme un des enjeux économiques majeurs de notre siècle, le Syntec Numérique encourage ses adhérents industriels à emprunter aux start-ups leurs méthodes !

« L'industriel peut s'inspirer des méthodes et pratiques des start-ups en matière de gestion de projet d'innovation : Lean Startup, agile, Design Thinking, TRIZ, etc. [...] La méthode TRIZ est un outil d'aide à la créativité adapté aux contextes techniques. Ces méthodes se prêtent bien aux tendances actuelles d'amélioration continue et de développement incrémental des produits. » [SYN 16].

Mais la start-up ne pourrait-elle pas s'inspirer des méthodes et pratiques éprouvées des grands groupes pour innover plus vite et plus facilement?

\subsection{TRIZ et start-up, par quoi commencer pour être pratique et opérationnel ?}

Pour s'inspirer des méthodes et pratiques éprouvées des grands groupes, les auteurs souhaitent rappeler les recommandations suivantes, issues de leur propre expérience :

- Commencer par «tuer le mythe » du génie faisant des affaires en cherchant l'idée géniale et inspirée ou l'évolution facilement copiable ou rapidement obsolète, mais utiliser une procédure robuste de créativité pour être prêt à « sauter sur la prochaine vague ».

- Ensuite, mettre en place le bon environnement d'affaires, traduire la vision stratégique du porteur de projet en cadencement et en diffusion, adapter un point de vue différent par une modélisation de l'idéal, mettre en relation les innovations avec la stratégie d'affaires en cohérence avec l'expérience client et accélérer la capacité de « serial innovation » en utilisant le modèle de la chaîne de valeur du système. En expliquant au jeune dirigeant les notions d'idéalité et de contradiction, lui permettre de garder en tête tout au long de son processus d'innovation que la chaîne de valeur de la start-up se situe au plus près du « résultat final idéal » malgré les aléas et les nécessités du quotidien. D'après l'expérience des auteurs, cet accompagnement pragmatique et efficace se fait avec le porteur de projet ou l'équipe dirigeante en une demi-journée, dès que la start-up est prête à se mettre en cause, à explorer son modèle d'affaires et à revisiter la solidité des idées, concepts ou prototypes, l'initiation à TRIZ étant implicite lors de l'usage des premiers outils et méthodes. L'aspect ludique fonctionne aussi très bien, auprès des « geeks » mais aussi dans les PME ou l'enseignement, avec des jeux de cartes basés sur TRIZ qui apportent une véritable amélioration de la créativité individuelle, de la synergie de groupe et de l'efficacité de l'équipe [LEP 14];

- Enfin, un suivi régulier (présentiel ou distant) permet de garder le cap, au-delà de la première idée, pour envisager et " planifier » les prochaines étapes (bien sûr adaptées le moment venu au contexte) dans une logique de pilotage efficace à moyen-terme et rassurante pour les investisseurs potentiels...

\subsection{Etude de cas : exemple d'usage de l'esprit start-up dans un groupe industriel}

Une entreprise industrielle du domaine de la défense souhaitait que l'association TRIZ France organise avec une école de gestion et d'ingénierie un atelier de deux heures pour innover avec des 
étudiants et des salariés de l'entreprise, sur le thème de l'informatisation de tâches pour des opérateurs, afin de ne plus utiliser de papier pour fournir les ordres de fabrication et les instructions des procédures.

Dans cette situation d'innovation «numérique » pour les opérateurs, il convenait de bien reformuler la question de la demande initiale de transformation en « fabrication intelligente » pour une entreprise ayant déjà des " solutions en tête ", avant de se lancer dans la résolution du " supposé problème » et d'étudier plusieurs hypothèses potentiellement contradictoires.

Une fois que la question a été présentée brièvement par le représentant de la société, quatre groupes de sept personnes ont été constitués, chacun animé par un expert de l'association TRIZ France ; avec le premier groupe, la réflexion a été concentrée, non pas sur la solution imaginée par le client, mais sur les questions exprimées comme : quelle est la meilleure façon d'intégrer les logiciels sur PC ou tablette fournis à l'opérateur?

\subsubsection{Outil "Cartographie du trajet"- description détaillée d'une journée typique, étape par étape}

Avec le premier outil, de type business ("cartographie du trajet"), les participants du premier groupe ont décrit leur vision de la vie de l'opérateur dans une journée typique, après avoir défini, avec l'aide du représentant de l'opérateur, le profil de la personne observée dans son travail quotidien. Ce profil a été décrit pas à pas au travers des activités de l'opérateur : toutes les informations que l'opérateur doit obtenir sont précisément notées ainsi que son ressenti sur son environnement de travail (ordinateurs, machines, collègues, ...). Ensuite, le groupe de travail a identifié les questions les plus importantes : obtenir le bon classeur pour la bonne procédure, lire, comprendre, mémoriser et appliquer les instructions de la procédure, réaliser l'autocontrôle du travail, enregistrer le travail comme terminé, puis revenir à la première étape pour l'action suivante.

La leçon apprise de ce premier atelier est qu'il est essentiel de mélanger dans le groupe de travail différents types de participants, des personnes expérimentées (salariés confirmés de l'entreprise) et des personnes « candides » (élèves-ingénieurs) afin de brasser les points de vue sans se concentrer trop rapidement sur un seul élément. Cela permet d'avoir une vue plus large des possibilités d'amélioration ou d'innovation et des ressources disponibles "libres" pour résoudre de façon inventive la situation initiale. Malgré la demande explicite de la grande entreprise de commencer tout de suite à travailler sur ses propres suggestions de solutions, cette démarche de type start-up a permis de « sortir du cadre » et de redéfinir « de bas en haut » les tâches de l'opérateur pour améliorer son travail, en ayant non seulement automatisé certaines de ses tâches avec une tablette ou un PC, mais en ayant surtout revu l'organisation de son travail en réordonnant des tâches ou en éliminant des gestes inutiles afin de redonner du sens à son travail quotidien.

\subsubsection{Outil "Visualisation TRIZ" - comprendre le vrai problème à résoudre et identifier les contradictions}

Avec le second outil, "Visualisation TRIZ", le premier groupe est passé à l'amélioration de la vie des opérateurs en cherchant maintenant réellement la solution « zéro papier » souhaitée pour soutenir le processus de fabrication. Il s'agissait simplement de photographier la situation existante, pour montrer la complexité qui serait atteinte en négligeant les caractéristiques du problème véritable : l'environnement complexe de la station de travail que l'opérateur doit apprendre à utiliser, plutôt que de se concentrer sur son propre travail. Il est ainsi apparu que la description détaillée du vrai problème à résoudre était : obtenir un classeur d'instructions, lourd, contenant plus de 200 pages, l'apporter à son poste de travail et le feuilleter longtemps avant de trouver le bon chapitre et les instructions à lire. Si l'opérateur se considère comme expérimenté, il ne va pas lire les instructions. Ainsi, si une instruction a changé, l'opérateur ne la lira pas et il se mettra lui-même ou il mettra l'efficacité de production dans une zone à risque. En utilisant la méthode TRIZ, la question a été décomposée en contradictions : le classeur doit être lourd et léger; contenir des informations détaillées accessibles rapidement mais lues 
sans effort. Ensuite l'étude des ressources à utiliser pour communiquer des instructions a été menée : enregistrement audio, voix humaine, schémas, phrases, mots, couleurs, dessins, vidéos, images. Les ressources les plus prometteuses ont été sélectionnées en fonction des types particuliers d'instructions et de la situation de l'opérateur dans l'usine. Plutôt que les phrases existantes, des illustrations ont été choisies en lien avec les typologies d'outils technologiques pour fournir leur statut (ressources "libres et/ou existantes") : il est apparu ainsi que les seules ressources nécessaires et suffisantes à la fourniture des instructions dont les opérateurs ont besoin sont l'écran existant, leur badge et leurs pieds.

La leçon apprise du second atelier est que la visualisation constitue un outil très puissant pour aider les participants à comprendre les caractéristiques sous-jacentes d'un objet ou d'un concept, afin de forger leur propre représentation mentale de cet objet dans la vie réelle.

\subsubsection{Finalement, en deux fois une heure, le premier groupe a été guidé par TRIZ «à son insu »!}

Avec le guide de résolution des contradictions pour soutenir le processus de pensée, l'équipe, hétérogène et non préalablement formée à la méthode TRIZ, a pu se concentrer efficacement sur la résolution de la situation proposée, non pas directement, mais après le détour par le "super-système » (journée de travail détaillée complète) puis le " sous-système " (visualisation des types de communication et d'illustration). Ce guidage implicite formel a aidé le groupe à ne pas diverger du véritable problème à résoudre et a facilité sa concentration sur sa résolution. De plus, il a aidé à démontrer pourquoi le problème existait et comment il était résolu en permettant la lecture inverse du processus de pensée.

Les trois autres groupes ont utilisé avec succès d'autres possibilités de la " boîte à outils TRIZ », mais la clause de confidentialité requise par la grande entreprise ne permet pas d'en dire davantage, sans dévoiler de secrets industriels.

\subsection{Start up et besoin de protection par la Propriété industrielle}

\subsubsection{Apport potentiel de TRIZ aux "jeunes pousses"}

Depuis plusieurs années, les auteurs s'interrogent sur ce que TRIZ pourrait apporter aux « jeunes pousses » (validation des premières idées, amélioration des concepts, renforcement de la protection) et ils ont présenté, dans une conférence internationale, l'état de leurs expérimentations chez un incubateur français pour former quelques start-ups à l'état d'esprit TRIZ [PRE 14].

Habituellement, la structure d'incubation consolide la validation du business plan avec les autres entrepreneurs et en fonction des revues des business angels, oriente la start-up vers les programmes publics de financement et prêts particuliers, facilite le " réseautage » avec ses pairs, organismes de financement et autres compétences techniques ou d'affaires disponibles dans écosystème de la start-up, ainsi qu'apporte le support administratif et logistique...

Pourtant, en plus de l'hébergement, accès Internet et autres services de base, il est rare pour ces jeunes entrepreneurs de bénéficier de services d'accompagnement utiles à leur développement et prochaine maturité, tels que l'aide à la prospective, la formation à la créativité, la pratique de la résolution de problèmes et l'initiation à la propriété intellectuelle...

\subsubsection{TRIZ, guide pratique d'innovation continue pour renforcer la PI des start-ups}

TRIZ pourrait apporter cette valeur différenciatrice aux incubateurs et à leurs start-ups incubées, pour les aider à comprendre ce qu'ils font (représentation systémique), leur donner une vision à moyen-terme (tendances d'évolution) et ainsi les orienter sur les aspects importants (résolution des contradictions) en termes d'innovation de marché, de produit ou de service : cette façon pragmatique de mutualiser l'apprentissage leur donnerait un avantage concurrentiel bien utile. Car le monde est rude pour une start-up : la pratique fréquente de l'innovation « ouverte» est devenue une chasse aux idées, 
que certains grands groupes s'approprient parfois sans respecter la PI des jeunes entreprises créatives, que celles-ci ont du mal à protéger, par manque de temps, d'argent ou de compétence, pour trouver la bonne façon de se protéger efficacement sans dépenser une fortune...

Dans le cas particulier qu'elle constitue, la « jeune pousse » doit-elle adopter une stratégie de type start-up (flexible et ouverte) ou plus classiquement de type industriel (contractualisation précise et protection solide), sachant que son « activité/produit/service » nécessite de nombreux partenariats avec des industriels parfois de taille mondiale, pour développer et mettre sur le marché sa solution générique et/ou unifiée?

Pourquoi ne pas utiliser les outils de la conception inventive pour résoudre cette contradiction extrême " se protéger sans se protéger », par exemple en essayant de soi-même contourner ses propres idées, de produire des concepts intégrant leur propre défense ou à l'inverse de publier rapidement pour empêcher tout brevet concurrent?

\subsubsection{Looky Lux, exemple lumineux d'une start-up, entre besoin d'ouverture et besoin de protection}

C'est la stratégie adoptée par la start-up « Looky Lux » accompagnée par un des co-auteurs qui l'a suivie pendant une année. Ce suivi est allé des premiers succès (Hackaton, deux fois primée ; Start-up Week-end, prix spécial du Jury; partenariat Orange, prêt de boîtiers connectés) aux difficultés de financement (manque de fonds d'amorçage), sans oublier les inévitables « pivots » nécessaires chaque trimestre dans la quête de son marché, les aléas (changements d'équipe informatique), les recherches de partenariats industriels et le pré-diagnostic INPI [SIR 16].

« Nous développons un système d'éclairage intelligent par le biais d'objets connectés : des boîtiers équipés de capteurs, qui viennent se brancher directement sur les lampadaires et communiquent vers un tableau de bord de télégestion du parc de lampadaires d'une ville », explique le porteur du projet [LOO $16]$.

Il s'agit d'adapter l'éclairage à des situations d'usage (route ou ville) afin de caractériser une situation de danger ou une zone d'ombre (jeux de lumières préprogrammés ou commandés) pour faciliter et sécuriser la circulation des véhicules et des piétons (cheminement nocturne, mise en garde lumineuse, lumière suiveuse ou "onde blanche"). Autrement dit, la finalité du projet consiste à développer et diffuser un objet connecté en open-hardware pour la gestion intelligente de l'éclairage et de l'infrastructure publique.

Quels sont donc les enjeux de Propriété Intellectuelle de cette start-up emblématique de ces entrepreneurs de la nouvelle économie, qui combine des éléments existants sur le marché (industriels ou gestionnaires de l'éclairage grand public,...) avec un mode de développement agile et participatif (partenariats locaux et stratégiques) tout en gardant secret le « coeur du système »? La réponse est donnée par l'INPI : « Ovni juridique, tout à la fois instrument et sujet de protection, l'objet connecté donne prise à des droits de propriété multiples qu'il convient d'appréhender de manière différenciée au sein d'une stratégie de protection globale et adaptée. » [INP 16].- «L'interopérabilité devient une exigence incontournable pour les industriels, fabricants de matériels et d'accessoires, les éditeurs, et les prestataires comme pour les clients et permet l'interface entre les différents systèmes. » [INP 16].

Dans son pré-diagnostic, l'INPI a pris en compte les paramètres suivants :

- Montage complexe de partenariats stratégiques et implication locale d'entreprises digitales (développement informatique, pour l'intelligence embarquée dans l'objet connecté, dans le Cloud pour le tableau de bord de télégestion et aussi applications sur smartphones pour les techniciens de maintenance ou certains usagers prioritaires) ; 
- Partenaires potentiels (fournisseurs de technologies et opérateurs industriels) : fabricant mondial de puce électronique, opérateur international de télécommunication, développement électronique et radio (technologie LoRaWAN), industriels dans plusieurs secteurs spécialisés...

Nous voyons que l'INPI a identifié plusieurs besoins/contraintes PI pour la start-up : relations déjà tissées avec des acteurs majeurs, sous-traitants ou partenaires stratégiques, réalisation d'un démonstrateur déployé pour l'éclairage public d'une commune, «intelligence réseau » (coeur de l'innovation et de la propriété intellectuelle), analyse «big data » et mise en forme de tableaux de bord. L'INPI a validé et a recommandé une combinaison de ses outils de protection du capital intellectuel : veille (recherches concurrentielles régulières via esp@cenet), datation (régulièrement effectuée depuis deux ans via une page dédiée sur Facebook), secret et confidentialité (contrat ponctuel via avocat d'affaires), pratiques contractuelles (à l'occasion via avocat d'affaires), dépôt de marques (autorisation "Lucky Luke" en cours), et dépôt de brevets (évaluation de l'automate connecté)...

Alors, la start-up a choisi le « respect des droits respectifs » en raison de l'imbrication de différents composants et la pluralité de créateurs susceptibles de revendiquer des droits sur son objet connecté ; de même, face à la prolifération de référentiels sectoriels et fermés, normes propriétaires payantes, trop coûteuses pour les PME, elle a fait le choix des systèmes ouverts (réseau LoRa).

\subsubsection{La démarche systématique proposée aux start-ups innovantes : "sortez couverts"!}

Dans les études de cas précédentes, les auteurs ont montré plusieurs façons d'accompagner le porteur de projet pour exprimer plus facilement son idée ou concept et en favoriser la production systématique des idées selon deux axes (améliorations successives et complexification technologique), même si elles ne seront exploitées que plus tard, lorsque la start-up en aura le temps et/ou que le marché sera prêt à les accueillir.

La démarche de conception inventive peut donc l'aider à établir une vision de moyen-terme pour cadencer subtilement ses innovations sur le marché en fonction de la maturité de son offre, de celle de ses concurrents et de l'envie de ses clients d'acheter ses produits ou services.

Le processus de conception inventive décrit dans les paragraphes précédents permet de renforcer la démarche générale en se protégeant de la concurrence sans nécessairement passer par des moyens juridiques, c'est-à-dire en faisant soi-même les tentatives de copie, contournement ou blocage qui arriveraient fatalement un jour, afin de découvrir par avance comment les concurrents actuels ou "disruptifs" pourraient porter préjudice à la start-up.

Avec l'arrivée prochaine de la normalisation des processus d'innovation, la question évoquée cidessus n'est plus de savoir s'il faut innover de façon systématique selon une démarche ayant prouvé son efficacité, mais quand cela sera-t-il "obligatoire" ou fortement recommandé pour se différencier dans un référentiel non seulement national ou européen mais de plus en plus mondialisé.

L'esprit start-up doit donc rester pionnier mais lucide sur son environnement et utiliser la conception inventive comme un processus à deux niveaux (stratégique et opérationnel) permettant d'évaluer les problèmes des clients et développer les solutions nouvelles dont ils ont besoin avec une capacité inventive décuplée et protégée méthodiquement.

« Il y a une chose bien plus grave que de ne pas avoir réussi ; c'est de ne pas avoir essayé. » (Franklin Roosevelt)

\section{Discussion sur le périmètre du présent article}

Les auteurs souhaitent souligner que le présent-article ne constitue qu'une petite partie des apports de TRIZ à un entrepreneur. Sur la base des formalisations méthodologiques qu'ils ont rappelées et forts 
de leur retour d'expérience, les auteurs sont convaincus-que le monde de la création d'entreprise doit se munir d'outils efficaces pour innover continuellement, car la route qui mène un créateur de start-up à sa réussite est souvent longue et semée de nombreuses embûches. Ils voient l'innovation continue comme une philosophie et comme un outil de travail à mettre en oeuvre de façon systématique et pas uniquement comme une résultante auto-satisfaisante de création d'offre de produit ou service à un instant $\mathrm{T}$.

Par son aspect structuré, partagé et forçant à la réflexion, TRIZ permet d'avoir un outil de travail universel (parmi d'autres) pour aborder les problèmes rencontrés dans le cadre de l'évolution d'une entreprise, qu'ils soient avec les clients, les investisseurs, les partenaires, les ingénieurs, les technologies...

Cet outil permet d'apprendre et de s'améliorer par un fonctionnement en étapes dont l'efficience peut être évaluée régulièrement et partagée avec d'autres dans un langage commun de démarche d'innovation. Enfin, TRIZ est un des rares outils « open-source », qui peut être adapté, enrichi, utilisé sans limite, où que ce soit dans le monde et quel qu'en soit sa destination commerciale ou non. Grâce à ce statut ouvert, la communauté qui s'approprie TRIZ est elle aussi ouverte, croît régulièrement dans toutes les régions du globe et tout particulièrement en Asie ces dernières années (Corée du Sud, Inde, Malaisie, Chine, Japon,...). C'est aujourd'hui une formidable opportunité pour partager innovations, cultures et marchés. Les auteurs souhaitent inviter les lecteurs à saisir cette opportunité et à grossir cette communauté en prenant part aux associations TRIZ dans le monde, qui sauront les aider à s'approprier ces techniques et outils, pour renforcer leur capacité à construire et protéger leur business.

\section{Conclusion}

Le présent article a instruit l'analyse d'une méthodologie apte à résoudre des problèmes techniques bloquant l'évolution fonctionnelle d'un objet technique, de telle sorte à faire progresser ce dernier vers l'idéalité par optimisations successives inscrites dans des trajectoires d'innovation. Cette méthodologie TRIZ a été située comme un phénomène de pensée technique né au milieu du $20^{\text {ème }}$ siècle, toujours d'actualité deux générations plus tard, consacré à l'approche méthodologique de l'invention technique envisagée comme une méthode de créativité dans le cadre de résolution inventive de problèmes techniques. Le socle de la méthode est constitué par des postulats de l'évolution des systèmes techniques, à laquelle est adossé le principe d'analogie utilisé par TRIZ. Celui-ci est mis en action au travers du repérage et de l'analyse des tendances d'évolution pour donner à ces derniers un sens prédictif au regard du déroulement du temps envisagé comme un élément de maturation fonctionnelle et technique. Des études de cas, dans un environnement de groupe industriel ou de start up, ont illustré les considérations formelles développées précédemment, en particulier pour une analyse approfondie consacrée au démarrage opérationnel d'une start up.

Tout au long du présent article, il a été donné à voir au lecteur la puissance d'une logique formelle de résolution de problème inscrite dans le prisme de l'évolution, participant d'une approche de recherche, d'une part, par abstraction, de généricité propre à engendrer par le principe d'analogie d'autres applications décontextualisées et d'autre part d'optimalité dans la mise en action. Ces deux fondamentaux caractérisent pleinement le domaine de la connaissance et il n'est pas surprenant que la construction empirique d'une telle méthodologie, très largement adossée aux principes fondateurs des théories de la connaissance ainsi qu'aux considérations usuelles des méthodes générales de résolution de problèmes, ait inspiré une théorisation ultérieure. Le succès du très large déploiement dans l'industrie tient donc, entre autres, à la simplicité des quelques raisonnements logiques à enchaîner, qui cachent à l'utilisateur la profondeur formelle des théories ultérieurement formulées qui les justifient.

De plus, le lecteur aura appris (ou retrouvé), par le cas concret de la start up cherchant à exister dans le monde complexe qui l'entoure, que le formalisme de logique de résolution de problème peut s'étendre à bon nombre des prises de décision du jeune entrepreneur : autrement dit que ce formalisme 
de cheminement mental est lui-même un état d'esprit, rigoureux et orienté vers la recherche de progrès, applicable par le même principe d'analogie que celui qui est au cœur de la méthode TRIZ.

Ingénieurs et hommes d'action, les auteurs du présent article sont beaucoup plus focalisés sur le coeur de la méthodologie et ses applications concrètes que sur la théorisation même que cette méthodologie a inspirée. Ils n'abordent donc pas les limitations mises au jour par cette théorisation qu'ils laissent aux chercheurs spécialistes du sujet.

Par ailleurs, le format de l'article n'a pas permis d'épuiser le sujet de l'apport potentiel de la méthode TRIZ aux entrepreneurs de start up, ce qui signifie bien sûr que les perspectives qu'il ouvre concernent l'élargissement de ce champ du potentiel à destination des jeunes pousses.

\section{Remerciements}

L'association TRIZ France remercie la revue "Technologie et Innovation" de l'avoir sollicitée pour illustrer les liens entre invention, innovation et propriété intellectuelle, ainsi que les experts de la Théorie de résolution des problèmes d'invention (TRIZ) qui témoignent de son utilisation pratique.

Les auteurs remercient leur collègue et directeur de publication invité de ce numéro spécial, Pierre Saulais, pour les conseils qu'il leur a prodigués.

\section{Bibliographie}

[ALT 06] ALTSHULlER, G., Et soudain apparut l'inventeur : les idées de TRIZ, Poitiers : Avraam Seredinsnsky, 2006.

[BER 16] BERTHOLET, G., Consulté le 2811 2016, sur http://www.guilhembertholet.com/, 2016

[BLA 16] BLANK, S., Consulté le 2911 2016, sur https://steveblank.com/2010/01/25/whats-a-startup-first-principles, 2016

[CAR 97] CARON, F., Les deux révolutions industrielles du XXème siècle, Paris : Albin Michel, 1997

[CAV 12] CAVALUCCI, D., « Une théorie de l'invention en support des activités R \& D : Outil et mise en oeuvre par l'exemple », Techniques de l'Ingénieur, 2012

[DUB 09] DUBOIS, S. I., "Comparison of non solvable Problem Solving Principles issued from CSP and TRIZ". Dans G. CASCINI, Computer-Aided Innovation (CAI) (Vol. 277), Springer, 2009

[FRI 16] FRIDENSON, P., Consulté le 2911 2016, sur 1001startups.f: http://1001startups.fr/dis-cest-quoi-une-start-up, 2016

[GUI 11a] GUILLOU, Y., La courbe en S, Consulté le 2211 2016, sur http://triz-experience.blogspot.fr/2011/04/lacourbe-en-s.html, 2011

[GUI 11b] GUILlOU, Y., Introduction aux lois d'évolution, Consulté le $25 \quad 112016$, sur http:/trizexperience.blogspot.fr/2011/12/introduction-aux-lois-devolution.html, 2011

[GUI 12] GUILlOU, Y., Le comment et le pourquoi, Consulté le $22 \quad 112016$, sur http://trizexperience.blogspot.fr/2012/06/le-comment-et-le-pourquoi.html, 2012

[GUI 14] GUILLOU, Y., L'évaluation au travers des lois d'évolution, Consulté le 22112016 , sur http://trizexperience.blogspot.fr/2014/08/levaluation-au-travers-des-lois.htm, 2014

[INP 16] INPI, La Propriété Intellectuelle et la transformation numérique de l'économie, Consulté le 22112016 , sur https:/www.inpi.fr/fr/services-et-prestations/etude-pi-et-economie-numerique

[LEP 14] LEPOT, X., NEVEUX, A., GUILLOU, Y., \& BAUDRUX, S., "2A2CI, an effective Innovation Methodology that makes TRIZ People \& Business compatible”, Global TRIZ Conference, Seoul, Korea, 2014

[LEU 07] LEUNG, W., \& YU, K., "Development of online Game-Based Learning for TRIZ". Dans K.-C. P.-K. HUI, Technologies for e-learning and Digital Entertainment (Vol. 4469), VOLUME 4469 OF LECTURE NOTES I: Springer, 2007 
[LOU 08] LOUAFA, T., \& PERRET, F.-L., Créativité et Innovation : L'intelligence collective au service du management de projet. LAUSANNE : Presses Polytechniques et Universitaires Romandes, 2008

[MAR 16] MARANZANA, S., CAILLAUD, E., \& ROSE, B., « La méthode d'aide à l'innovation TRIZ et les limites de son déploiement en entreprise », CONFERE'16, Prague, 2016

[MEY 07] MEYLAN, C., Système de stimulation de la créativité et d'aide à l'innovation, méthodes pratiques pour la résolution de problèmes techniques et la recherche de nouvelles opportunités d'affaires, TEXTO, 2007

[PRE 14] PREVOST, E., BRUNO, G., GUILLOU, Y., SIRE, P., \& CONRARDY, C., "WhatT roles can TRIZ play in Start up incubators?" Global TRIZ Conference, Séoul, 2014.

[RIE 12] RIES, E., The Lean Startup, Pearson, 2012

[RIW 13] RIWAN, A., "TRIZ helps blind people to find their ways in subway stations”, TRIZ Future Conference, Paris, France, 2013

[RIW 14] RIWAN, A., “Enhanced Experience and Safety in interactive Robots”, Global TRIZ Conference, Seoul, Korea, 2014

[RIW 15] RIWAN, A., » TRIZ ou l'aide à la conception inventive : comment j'ai imaginé mon robot », Consulté le 2211 2016, sur CEA :

http://www.list.cea.fr/images/stories/recherche_technologique/vie_scientifique/seminaires_12.0/10_09_2015_alain_ri wan_triz_ou_1_aide_a_la_conception_inventive.pdf, 2015

[SAU 13] SAULAIS, P. Application de la gestion des connaissances à la créativité des experts et à la planification de la R\&T en milieu industriel de haute technologie. Thèse de doctorat, Telecom Ecole de Management, 2013

[SER 11] SEREDINSKI, A., \& JADAUD, D., « TRIZ : les notions de base », Revue Technologie (172), 2011

[SIR 16] SIRE, P., « Objets connectés pour systèmes d'éclairage intelligent - LOOKY LUX, gestion proactive de la lumière », Forum Intelligence économique et stratégique, Rouen, 2016

[SYN 16] SYNTEC, Transformer l'industrie par le numérique. Consulté le 2211 2016, sur http://www.syntecnumerique.fr/actualite/transformer-lindustrie-numerique, 2016

[TRE 12] TRENDEMIC, Consulté le 2911 2016, sur http://trendemic.net/esprit-start-up.htm, 2012

[TR1 16a] TRIZ-France, Consulté le 2211 2016, sur http://www.trizfrance.org, 2016

[TR1 16b] TRIZ-Wikipedia, Consulté le 2211 2016, sur https://fr.wikipedia.org/wiki/triz, 2016

[USI 03] USINE-NOUVELLE. "Découvrir la méthode TRIZ”, Consulté le $22 \quad 11$ 2016, sur http://www.usinenouvelle.com/article/decouvrir-la-methode-triz.N5956, 2003

[VOI 12] VOISEMBERT, S., Conception et modélisation d'un bras d'inspection robotisé ultra léger, Thèse de doctorat, CNAM, 2012.

[WIK 16] WIKILEAN. Consulté le 2211 2016, sur http://www.wikilean.com/Articles/LeanOvation/3-Les-outils-de-lacreativite-6-articles/TRIZ, 2016

[WIP 16] WIPO,Consulté le 2211 2016, sur http://www.wipo.int/sme/fr/documents/patent_information.htm, 2016 